\title{
Pixel sensors with different pitch layouts for ATLAS Phase-II upgrade
}

\section{Paul.Dervan@cern.ch*}

Different pitch layouts are considered for the pixel detector being designed for the ATLAS upgraded tracking system which will be operating at the High Luminosity LHC. The tracking performance in the Endcap pixel regions could benefit from pixel layouts which differ from the geometries used in the barrel region. Also, the performance in different barrel layers and eta regions could be optimised using different pixel sizes. This presentation will report on the development and tests of pitch layouts which could be readout by the FE-I4 ASICs. The pixel geometries include $50 \times 250 \mu \mathrm{m}^{2}, 25 \times 500 \mu \mathrm{m}^{2}, 100 \times 125 \mu \mathrm{m}^{2}, 125 \times 167 \mu \mathrm{m}^{2}, 50 \times 2000 \mu \mathrm{m}^{2}$ and $25 \times$ $2000 \mu \mathrm{m}^{2}$. The sensors with geometries $50 \times 250 \mu \mathrm{m}^{2}, 25 \times 500 \mu \mathrm{m}^{2}$ and $100 \times 125 \mu \mathrm{m}^{2}$ were irradiated and tested at the DESY testbeam. These and other testbeam results as well as results from characterisation of these sensors in the laboratory will be presented.

Technology and Instrumentation in Particle Physics 2014,

2-6 June, 2014

Amsterdam, the Netherlands

${ }^{*}$ Speaker. 


\section{Introduction}

In approximately 2024, the Large Hadron Collider (LHC) will be upgraded to the High Luminosity LHC (HL-LHC). The upgrade is foreseen to increase the LHC design integrated luminosity by a factor ten. The ATLAS [1] experiment will need to build a new tracker for HL-LHC operation, which needs to be suited to the harsh HL-LHC conditions in terms of particle rates and radiation doses. In order to cope with the increase in pile-up backgrounds at the higher luminosity, an all-silicon sensor detector is being designed.

The baseline layout [2] for the pixel upgrade forsees the sensors on the inner barrel layers to be readout by a new chip, with a pixel size of $50 \times 50 \mu \mathrm{m}^{2}$, and the sensors on the outer barrel and endcap layers/disks to be readout by the FE-I4 chip [3] with a pixel size of $250 \times 50 \mu \mathrm{m}^{2}$. In order to study the pitch of the inner barrel layer, $500 \times 25 \mu \mathrm{m}^{2}$ and $125 \times 100 \mu \mathrm{m}^{2}$ geometry sensors can be used. The $500 \times 25 \mu \mathrm{m}^{2}$ geometry is also investigated for use for the higher eta regions on the outer barrel layers, where the increased incidence angle will increase the cluster size for the normal $250 \times 50 \mu \mathrm{m}^{2}$ sensor. Square modules $\left(125 \times 100 \mu \mathrm{m}^{2}\right.$ and $\left.167 \times 125 \mu \mathrm{m}^{2}\right)$ are being investigated for the endcap where demands on the $\phi$ and $r$ resolution are very different from the barrel.

\section{Sensors}

In partnership with Micron Semicondutor Ltd [4], we have developed various wafers of pixel detectors. The CERN pixel $\mathrm{V}$ wafer was designed to study different pixel geometries. The different pixel geometries on the CERN pixel V wafer are: $125 \times 100 \mu \mathrm{m}^{2}$ (square) with 26880 pixels, 167 $\times 125 \mu \mathrm{m}^{2}$ (square) with 15680 pixels, $250 \times 50 \mu \mathrm{m}^{2}$ (current baseline for outer barrel pixel layers) with 26880 pixels, $500 \times 25 \mu \mathrm{m}^{2}$ (elongated) with 26880 pixels, $2000 \times 25 \mu \mathrm{m}^{2}$ (strixel) with 6720 pixels and $2000 \times 50 \mu \mathrm{m}^{2}$ (strixel) with 3660 pixels. The CERN pixel V wafer also contains sensors with both DC and AC coupling to allow future performance studies comparing the two options. They have been produced with thickness of $300 \mu \mathrm{m}, 200 \mu \mathrm{m}, 150 \mu \mathrm{m}$ and $100 \mu \mathrm{m}$. The results presented here are for sensors with DC coupling and thickness of $300 \mu \mathrm{m}$ and have a full depletion voltage of $80 \mathrm{~V}$. The sensors under test were bump bonded to FE-I4 chips at Advacam [6]

\section{Test Beam Results}

The DESY test beam consisted of $4 \mathrm{GeV}$ positrons [7]. The tracking was performed by the ACONITE beam telescope (a copy of the EUDET beam telescope [8]). The Devices Under Test (DUTs) are mounted inbetween the two arms of the telescope). Only two DUTs were mounted due to the multiple scattering of the $4 \mathrm{GeV}$ beam. The sensitive elements of the telescope planes are Mimosa26 [9] active pixel sensors with a pixel pitch of $18.4 \mu \mathrm{m}$. Each plane consists of 1152 $\times 576$ pixels covering an active area of $21.2 \times 10.6 \mathrm{~mm}^{2}$. The tracking resolution is estimated to be $2 \mu \mathrm{m}$ [10]. The trigger was provided by four scintillators (two up and two down stream of the telescope), which resulted in an effective sensitive area of $2 \times 1 \mathrm{~cm}^{2}$. The trigger rate was $1 \mathrm{kHz}$. The hit efficiency was measured by matching hits on the sensors to a matching track in the telescope. 
Various comparisons were carried out, for example between the $250 \times 50 \mu \mathrm{m}^{2}$ and the $500 \times$ $25 \mu \mathrm{m}^{2}$ non-irradiated sensors, and between non-irradiated and irradiated sensors. The irradiations were carried out at the Birmingham irradiation facility [5], to a fluence of $1 \times 10^{15} 1 \mathrm{MeV} \mathrm{n}_{e q} \mathrm{~cm}^{-2}$. The results will be presented in the following sub-sections.

\subsection{Comparison between the $250 \times \mathbf{5 0} \mu \mathrm{m}^{2}$ sensor vs $500 \times 25 \mu \mathbf{m}^{2}$ sensor}

Tracks are generated when there are at least 4 out of 6 hits in the telescope planes and at least one in the DUT. Tracks extrapolated from telescope match to a hit if the hit and extrapolated impact point on the DUT containing the hit are closer than $400 \mu \mathrm{m}$ in the long direction and $150 \mu \mathrm{m}$ in the short direction. The hit efficiency for both sensors was shown to be uniform over their area and greater than $99.5 \%$, for both thresholds studied (3200e and 1600e).

Figure 1 shows the measured residuals for the $250 \times 50 \mu \mathrm{m}^{2}$ and the $500 \times 25 \mu \mathrm{m}^{2}$ devices at a threshold of 3200e; in $\mathrm{x}$ (long pixel direction) the distribution is flat and centred on zero with the RMS value well approximated by pitch $\sqrt{(12)}$ (for the $500 \times 25 \mu \mathrm{m}^{2}$ device we expect 144.3 $\mu \mathrm{m}$ and $7.2 \mu \mathrm{m}$ in $\mathrm{x}$ and $\mathrm{y}$. And for the $250 \times 50 \mu \mathrm{m}$ we expect $72.2 \mu \mathrm{m}$ and $14.4 \mu \mathrm{m}$ in $\mathrm{x}$ and $\mathrm{y}$ ). In $y$ the residual is improved by the smaller pixel size.
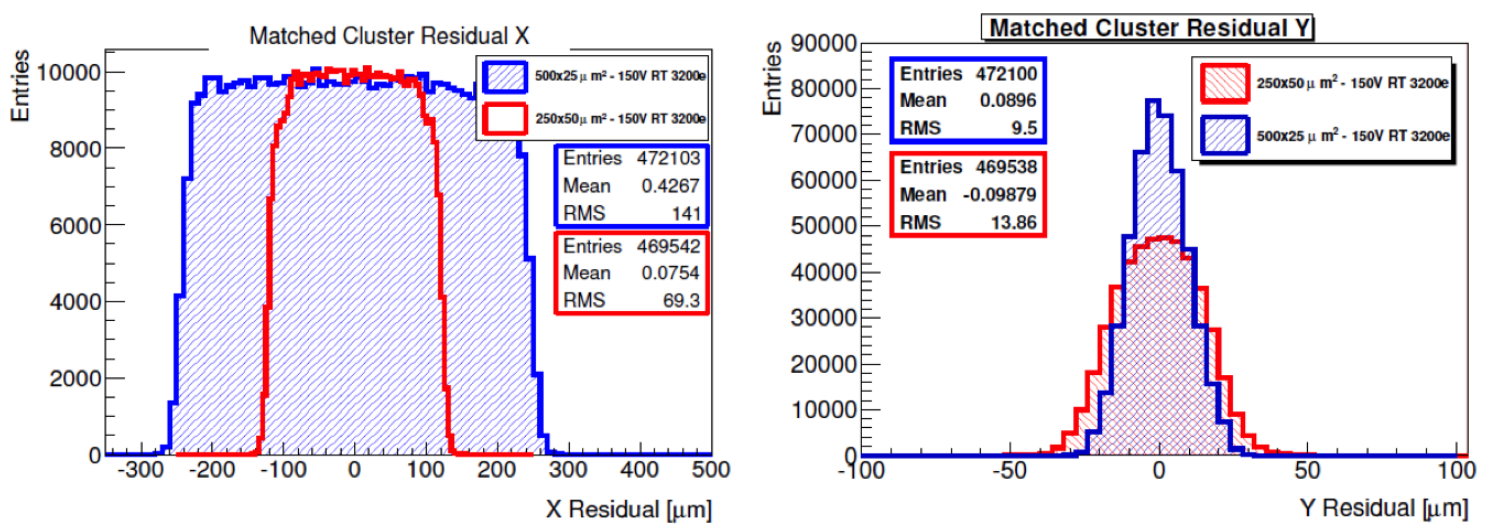

Figure 1: The measured residuals in $\mathrm{x}$ (left) and $\mathrm{y}$ (right) for the $250 \times 50 \mu \mathrm{m}^{2}$ and the $500 \times 25 \mu \mathrm{m}^{2}$ devices at a threshold of 3200e.

Figure 2 shows that there is a small difference in the maximum time over threshold as measured for the $250 \times 50 \mu \mathrm{m}^{2}$ and the $500 \times 25 \mu \mathrm{m}^{2}$ devices at a threshold of $1600 \mathrm{e}$ and $3200 \mathrm{e}$, due to the difference in tuning and the effect of the different cluster size.

Figure 3 shows the cluster size in $\mathrm{x}$ and $\mathrm{y}$ for the two devices. It is clearly seen that there is negligible change in the cluster size in going from the $250 \times 50 \mu \mathrm{m}^{2}$ and the $500 \times 25 \mu \mathrm{m}^{2}$ in $\mathrm{x}$, whilst there is an increase in the charge sharing in the y when going from 50 to $25 \mu \mathrm{m}$.

\subsection{Comparing a $125 \times 100 \mu \mathrm{m}^{2}$ non-irradiated device with an irradiated one $\left(1 \times 10^{15}\right)$}

The modules were glued down onto low mass $400 \mu \mathrm{m}$ thick carbon fibre plates to reduce multiple scattering but still provide an efficient cooling path. The cooling was provided by dry ice (module temperatures were $\approx-25^{\circ} \mathrm{C}$ ). Figure 4 shows the hit efficiency for the non-irradiated $(100 \mathrm{~V}$ bias voltage, its full depletion voltage is $\approx 80 \mathrm{~V}$ ) and irradiated sensor (500V bias voltage, 


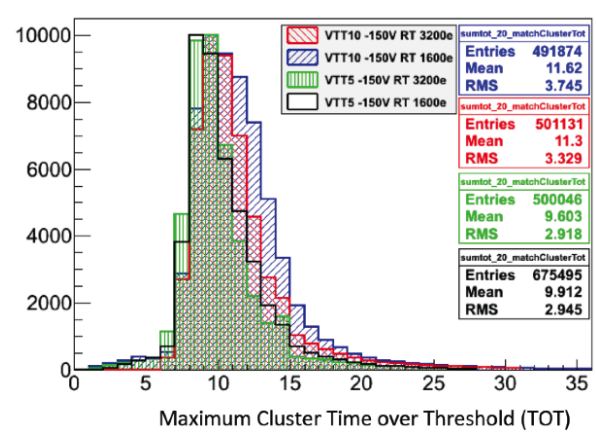

Figure 2: The maximum time over threshold as measured for the $250 \times 50 \mu \mathrm{m}^{2}$ and the $500 \times 25 \mu \mathrm{m}^{2}$ devices at thresholds of $1600 \mathrm{e}$ and $3200 \mathrm{e}$.
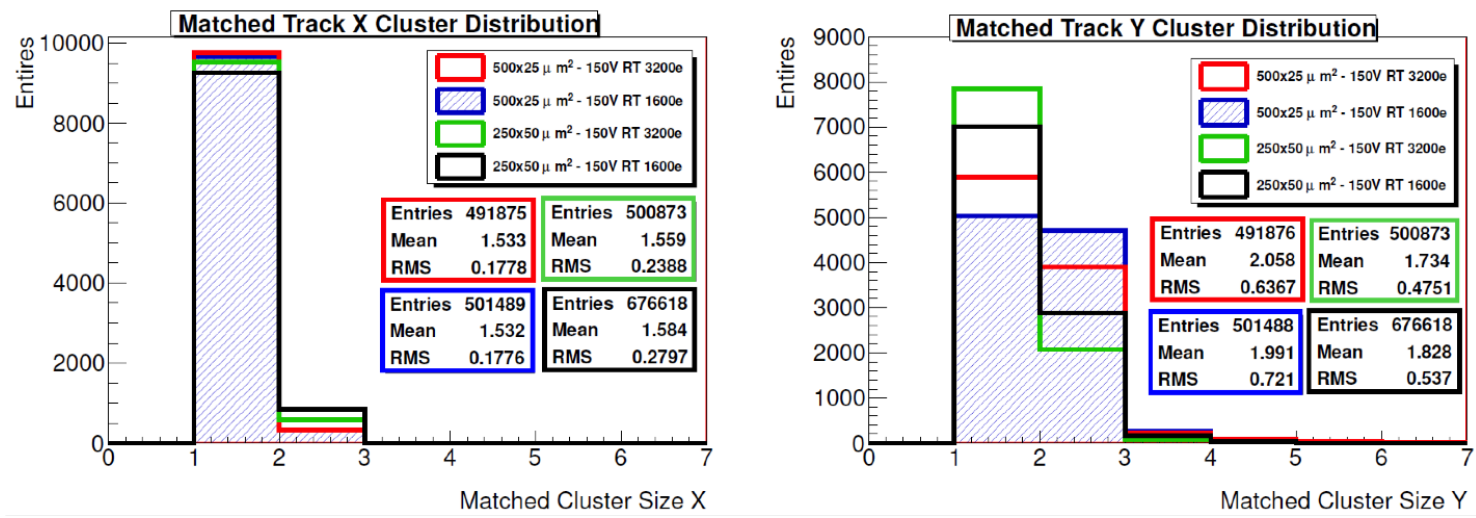

Figure 3: The cluster size in $\mathrm{x}$ (left) and $\mathrm{y}$ (right) as measured for the $250 \times 50 \mu \mathrm{m}^{2}$ and the $500 \times 25 \mu \mathrm{m}^{2}$ devices at thresholds of $1600 \mathrm{e}$ and $3200 \mathrm{e}$.

with a full depletion voltage of $\approx 600 \mathrm{~V})$. As can be seen the efficiency is better than $99 \pm 0.03$ $\%$ and is uniform over the sensor. The irradiated sensor has more masked channels $(10 \%$ of the channels compared to $1 \%$ for a non-irradiated sensor).

Figure 5 shows the hit efficiency, with a threshold of 2000e, as a function of bias voltage (excluding masked pixels). As expected we get full efficiency for the non-irradiated module, but we need a higher bias voltage for the irradiated sensor.

Inner pixel efficiency is also of interest as we have the punch through bias for the pixels at the edge. The efficiency of a pixel is plotted using the extrapolated hit position. All hits are plotted across the smallest repeatable regular structure which is four pixels. Figure 6, shows this for the $125 \mu \mathrm{m} \times 100 \mu \mathrm{m}$ sensor. All hits are then folded into a single pixel which is represented by the green box in Figure 6. As a result the punch through bias structure will be replicated twice. The method of folding used means that punch through bias structures will be in the right side corners of the inner pixel efficiency plots. Any drop in efficiency in the left side is due to charge sharing between the corners of the pixel implants. As expected the efficiency for the non-irradiated module is high, $99.9 \pm 0.03 \%$ at $100 \mathrm{~V}$. Where as the efficiency changes with bias voltage for the irradiated sensor, Figure 7. Inefficient regions due to charge sharing between neighbouring pixels quicly 


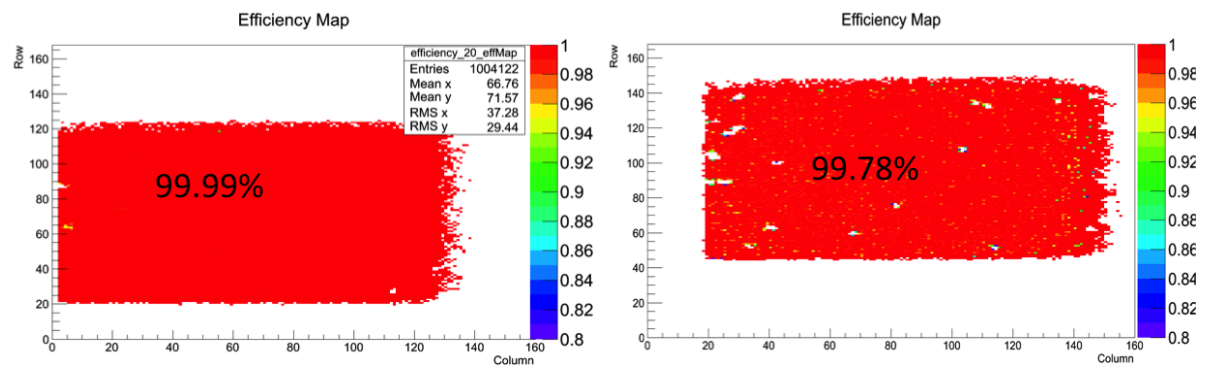

Figure 4: The efficiency map for the non-irradiated (left) and irradiated (right) $125 \times 100 \mu \mathrm{m}^{2}$ modules at 100 and $500 \mathrm{~V}$ respectively.

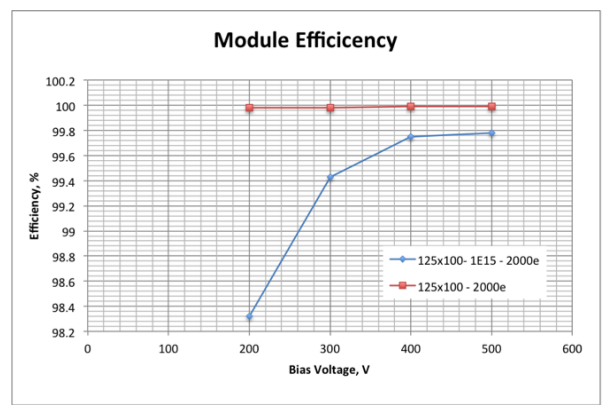

Figure 5: The hit efficiency as a function of bias for the non-irradiated (red) and irradiated (blue) $125 \times 100$ $\mu \mathrm{m}^{2}$.

disappears, while the inefficient region due to the punch through biasing structures only decreases in size.

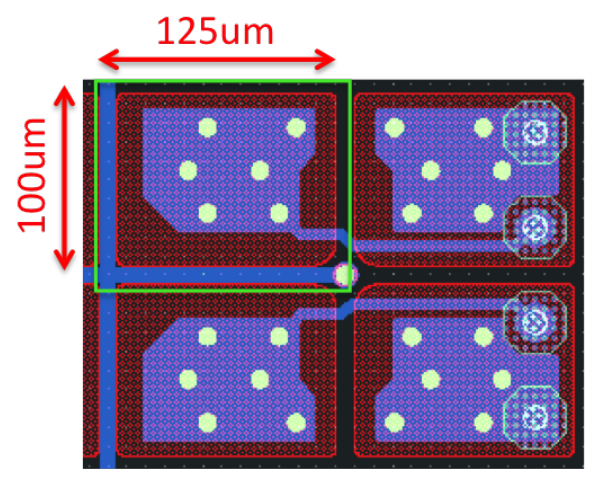

Figure 6: A schematic of four pixels, showing the bias dot in the middle.

\subsection{Comparing a $250 \times 50 \mu \mathrm{m}^{2}$ non-irradiated sensor with an irradiated one $\left(1 \times 10^{15}\right)$}

These modules were tested in the same maner as the $125 \times 100 \mu$ m modules. The overall efficiency for the non-irradiated module was $99.99 \pm 0.03 \%$ at $100 \mathrm{~V}$ and $99.32 \pm 0.03 \%$ at $500 \mathrm{~V}$ 

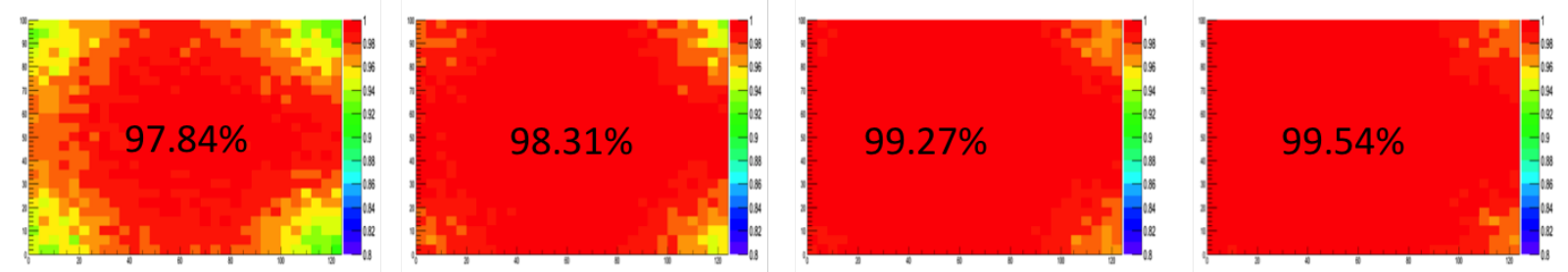

Figure 7: The inner-pixel hit efficiency as a function of bias (from left to right, 200V, 300V, 400V and $500 \mathrm{~V})$ for the irradiated $125 \times 100 \mu \mathrm{m}^{2}$.

for the irradiated module. Figure 8 shows the efficiency as a function of bias voltage for the two sensors.

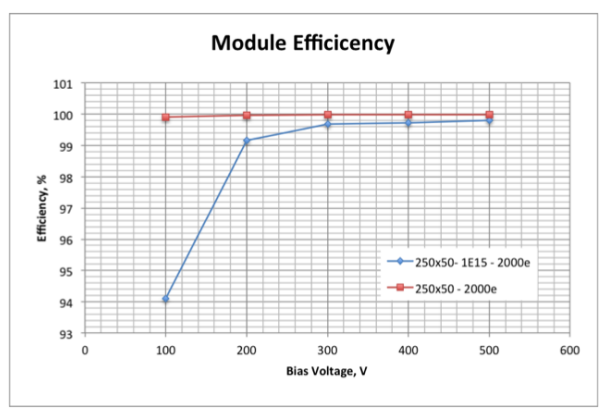

Figure 8: The hit efficiency as a function of bias for the non-irradiated (red) and irradiated (blue) $250 \times 50$ $\mu \mathrm{m}^{2}$.

The resolution within pixel's results were similar. For the non-irradiated module it is $99.99 \%$ at $100 \mathrm{~V}$, whereas the irradiated modules efficiency changes as a function of bias voltage, Figure 9. The effect of the punch through biasing can be seen on the right side of the pixel.

\section{Summary}

Pixel sensors with geometries that include $50 \times 250 \mu \mathrm{m}^{2}, 25 \times 500 \mu \mathrm{m}^{2}, 100 \times 125 \mu \mathrm{m}^{2}$, $125 \times 167 \mu \mathrm{m}^{2}, 50 \times 2000 \mu \mathrm{m}^{2}$ and $25 \times 2000 \mu \mathrm{m}^{2}$, have been fabricated by Micron. These have been bump bonded to FE-I4 readout chips. Some of them have been irradiated to $1 \times 10^{15}$ $\mathrm{n}_{e q} \mathrm{~cm}^{-2}$ and then tested in test beams at DESY with $4 \mathrm{GeV}$ electrons. The tracking efficiency has been shown to be excellent in all cases.

\section{References}

[1] G. Aad et al, The ATLAS experiment at the CERN Large Hadron Collider, JINST 3 (2008) S08003.

[2] Letter of Intent for the Phase-II Upgrade of the ATLAS Experiment, Technical Report CERN-LHCC-2012-022. LHCC-I-023, CERN, Geneva, 2012.

[3] M. Garcia-Sciveres et al., The FE-I4 pixel readout integrated circuit, Nucl. Instr. Meth. A636, S155-S159 (2011). 
[4] Micron Semiconductor Ltd., http://www.micronsemiconductor.co.uk

[5] P. Dervan et al, The Birmingham Irradiation Facility, Nucl. Instr. Meth. A730, 101-S104 (2013).

[6] www.advacam.com

[7] http://adweb.desy.de/home/testbeam/WWW/Description.html

[8] A. Bulgheroni et al., Results from the EUDET telescope with high resolution planes, Nucl. Instr. Meth. A623, 399-401 (2010).

[9] C. Hu-Guo, A ten thousand frames per second readout MAPS for the EUDET beam telescope, CERN-2009-008.

[10] J. Behr, Jets at high $Q^{2}$ at HERA and Test Beam Measurements with EUDET Pixel Telescope, Dissertaion and Ph.D thesis (2010) - ZEUS, Universitat Hamburg, Chapter 16. 

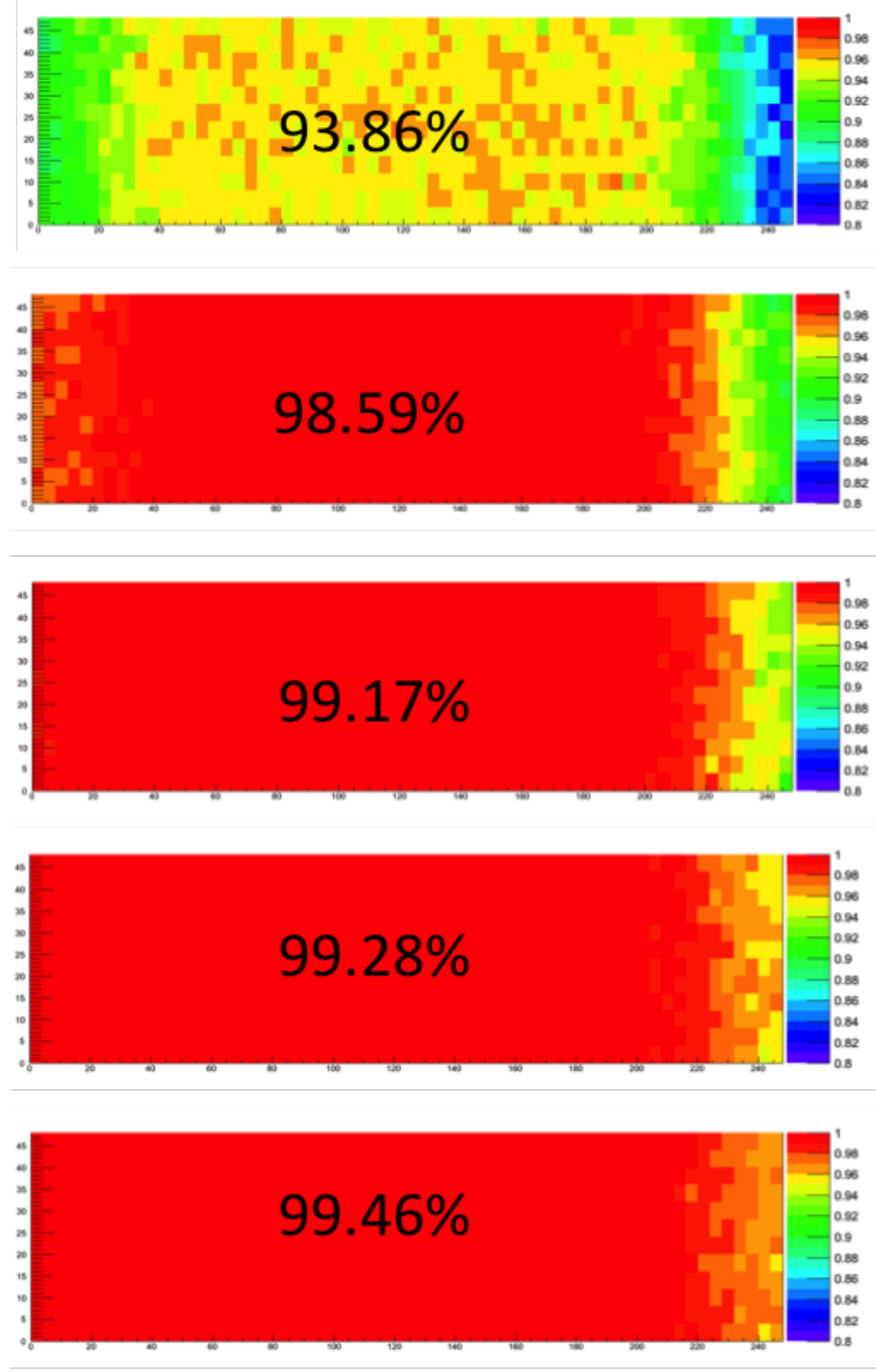

Figure 9: The inner-pixel hit efficiency as a function of bias (from top to bottom, 100V, 200V, 300V, 400V and $500 \mathrm{~V}$ ) for the irradiated $250 \times 50 \mu \mathrm{m}^{2}$. 